\title{
Statistisch signifikant - aber auch für die Praxis bedeutsam?
}

\author{
WISSENSCHAFT ERKLÄRT: KLINISCHE SIGNIFIKANZ Oft sprechen Wissenschaftler \\ von signifikanten Ergebnissen in ihren Studien. Statistisch oder klinisch signifikant? \\ Die Bedeutung dieser Begriffe und ihren Bezug zum physiotherapeutischen Praxis- \\ alltag beschreibt dieser Artikel.
}

\section{D}

er Großvater von Lars ist seit einigen Jahren an Morbus Parkinson erkrankt. Seine Hände zittern, und er geht immer langsamer. Lars ist vor allem aufgefallen, dass sein Großvater immer kleinere Schritte macht und zwischendurch „stecken“ bleibt.

Eine groß angekündigte Studie verspricht nun eindeutige Verbesserungen für Patienten mit Parkinson. Sie wirbt damit, dass sich bei der untersuchten Therapiemethode vor allem das Gangbild der Patienten signifikant verbessert. Das macht Lars neugierig, und er liest die Studie mit großem Interesse. Er kämpft sich durch den Methodenteil, aber die Ergebnisse versteht er nicht. Er fragt sich, was genau eine statistisch signifikante Verbesserung der Schrittlänge ist. Die Wissenschaftler schlussfolgern in ihrer Studie, dass ihr neuer Ansatz äußerst vielversprechend ist und sich dadurch die Schrittlänge von Menschen mit Parkinson statistisch signifikant verbessert. Lars ist von der korrekt erscheinenden und vor allem wissenschaftlich dargelegten Methodik ziemlich beeindruckt. Aber als er sich die angeblich so signifikante Verbesserung der Schrittlänge ansieht, wundert er sich: Die Schritte der teilnehmenden Patienten haben sich um nur $2 \mathrm{~cm}$ verlängert. Können $2 \mathrm{~cm}$ für den Alltag von Patienten signifikant besser sein?

\section{Es gibt zwei Arten von Signifikanz > Lars} hinterfragt den Begriff der Signifikanz - oder auch Bedeutsamkeit. Er findet heraus, dass es zwei Arten von Signifikanz gibt: die statistische und die klinische Signifikanz. Die statistische Signifikanz wird mathematisch berechnet. Wenn in einer Studie alle Patienten zum Beispiel eine Schrittlänge von $20 \mathrm{~cm}$ haben, und ihre Schritte am Ende einer Therapie $22 \mathrm{~cm}$ lang sind, spricht man von einer statis-

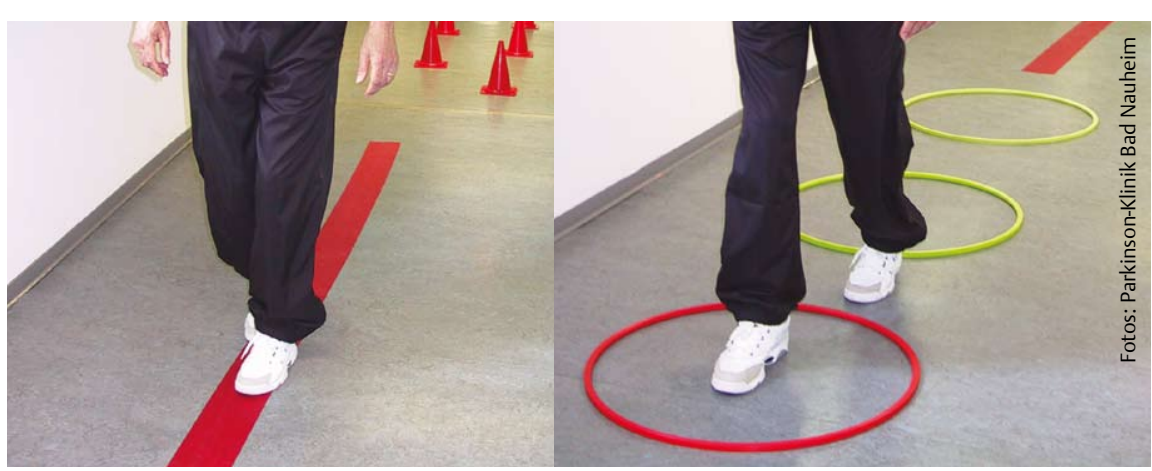

Die klinische Signifikanz bestimmt, ob eine durch Training erreichte größere Schrittlänge für Patienten im Alltag relevant ist oder nicht.

tischen Signifikanz. Die klinische Signifikanz ist weitaus schwieriger zu bestimmen, weil man sie nicht einfach berechnen kann. Sie drückt nämlich aus, wie bedeutsam ein Studienergebnis im klinischen Alltag tatsächlich ist. Der eine Patient hält eine Verlängerung seiner Schritte um $2 \mathrm{~cm}$ für einen kleinen Gewinn, da seine eigentliche Schrittlänge nur noch $9 \mathrm{~cm}$ betrug und er sich hauptsächlich im Haus aufhält. Der andere findet $2 \mathrm{~cm}$ ein eher enttäuschendes Ergebnis, da er trotz der Verbesserung noch immer nicht sein gewünschtes Ziel erreicht - einen Spaziergang im Grünen machen zu können.

\section{Signifikanz ist subjektiv > Diese Gegensätze} findet man auch in der Physiotherapie. „Klinisch bedeutsam“ kann für Physiotherapeuten völlig Unterschiedliches bedeuten. Beim Beispiel von Lars ist es relativ einfach. Wenn die Schritte des Großvaters lediglich $2 \mathrm{~cm}$ länger werden, wird ihm das nicht wirklich bedeutsam erscheinen. Aber wo ist die Grenze? Bei $5 \mathrm{~cm}$, bei $10 \mathrm{~cm}$ ? Dies kann niemand verbindlich festlegen. Und das ist das Schwierige an der klinischen Signifikanz.
Doch wie kommt man aus diesem Dilemma heraus? Zum einen sollten Wissenschaftler in ihren Veröffentlichungen nicht nur erwähnen, ob ein Ergebnis statistisch signifikant ist, sondern auch, inwiefern es klinisch signifikant sein könnte. Zum anderen sollten sich Leser immer fragen, welche direkte Bedeutung ein Studienergebnis für ihre Patienten in der Praxis haben kann. Es wäre wünschenswert, dass Forscher bereits vor Beginn ihrer Studie protokollieren, welches erzielbare Ergebnis tatsächlich eine klinische Bedeutsamkeit aufweist.

Signifikanzniveau vorher festlegen > Physiotherapeutisches Fachwissen bestimmt die klinische Signifikanz. Forscher sollten sich deshalb Folgendes fragen: Für welchen Patienten soll eine Behandlung klinisch signifikant sein? Und in welchem Maße?

Lars weiß nun, dass das Ergebnis dieser Studie für seinen Großvater nicht bedeutsam ist. Aber er hat auch verstanden, dass die einfache und oftmals gestellte Frage, ob ein Studienergebnis klinisch signifikant ist, extrem schwierig und nicht einheitlich zu beantworten ist.

Prof. Dr. Jan Mehrholz 